\title{
Mediation of Electrostatic Discharge Induced Morphological Damage in Atomically Precise Tips
}

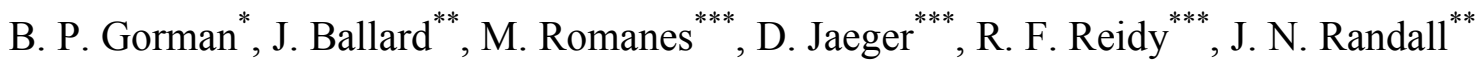 \\ *Department of Metallurgical and Materials Engineering, Colorado School of Mines, Golden, CO \\ 80401 \\ **Zyvex Laboratories, Inc., Richardson, TX \\ ***Department of Materials Science and Engineering, University of North Texas, Denton, TX \\ 76208
}

Tip geometry can have a large impact on image quality, resolution, and, depending on the sensing mode, image interpretation of scanned probe microscopy data [1]. Until recently, SPM investigations have lacked detailed characterization of tip geometries. For instance, an in-depth analysis of the tip geometry could lead to standards-based tip imaging via envelope functions [2]. Similar to SPM, atom probe tomography and field ion microscopy could also benefit from a better understanding of tip geometries for improved reconstruction accuracy, evaporation potential determination, and trajectory aberration correction. Successful atomically precise manufacturing (APM) using STM tips depends heavily on the ability to develop and characterize tips with near atomic surface precision [3].

During development of tips for APM, several processing techniques for extremely low radius of curvature $\mathrm{W}$ tips were developed. In order to fully characterize the tip geometry, hardware was developed that allows for cross sectional tomographic imaging of $\mathrm{W}$ wire shaped tips in a modern TEM or STEM, along with FIM, Atom Probe Tomography, and imaging in a UHV STM [4]. During characterization of the tip geometries using SEM, TEM, FIM, and atom probe, it was found that the tip radius was several orders of magnitude larger than expected (Fig 1). Characterization of the tips using STEM-EELS, -EDS and atom probe tomography revealed the presence of a thick $(>50$ $\mathrm{nm}$ ), low density $\mathrm{WO}_{\mathrm{x}}$. The sub-oxide layer was significantly thicker and less dense than expected for simple reactions with air.

Subsequent changes in the experimental methods for cross-examination with all of the above techniques revealed that the oxide formation was actually due to electrostatic discharge (ESD). It is worth noting that most ESD events that result in tip morphology changes are not observable visually or via human touch. Using good ESD tip handling protocol such as continuous grounding of specimen holders and tweezers during transfer had a profound effect on the ability to image in STM mode with nearly atomically sharp tips (Fig 2). Correlating the tip geometry with STM operation has also been illustrated in this work.

References

[1] J. S. Villarrubia, J. Res. Nat. Inst. Stand. Technol., 102, 425 (1997).

[2] D. J. Keller and F. S. Franke, Surf. Sci., 294, 409 (1993).

[3] J. N. Randall, et.al., J. Vac. Sci. Tech. B, 27, 2764 (2009).

[4] B. P. Gorman, et.al., Microscopy Today, 16, 42 (2008).

[5] Program supported by DARPA-MTO under the Tip Based Nanofabrication program managed by Tom Kenny contract N66001-08-C-2040. 


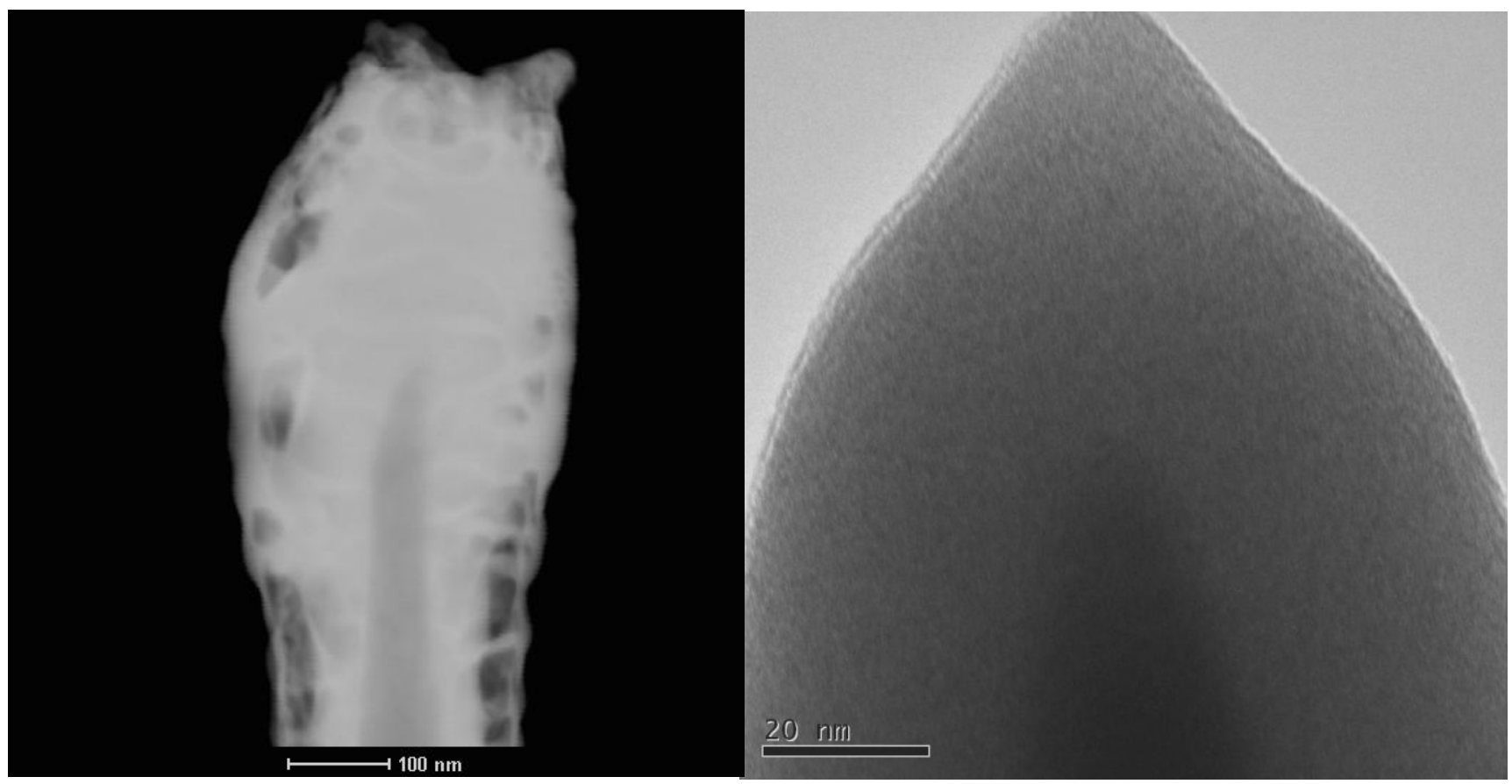

FIG. 1. Representative (a) STEM-HAADF and (b) bright field TEM images of improperly handled $\mathrm{W}$ tips. The layer surrounding the $\mathrm{W}$ core consists of an amorphous, porous $\mathrm{WO}_{\mathrm{x}}$ material deposited through an electrostatic discharge.
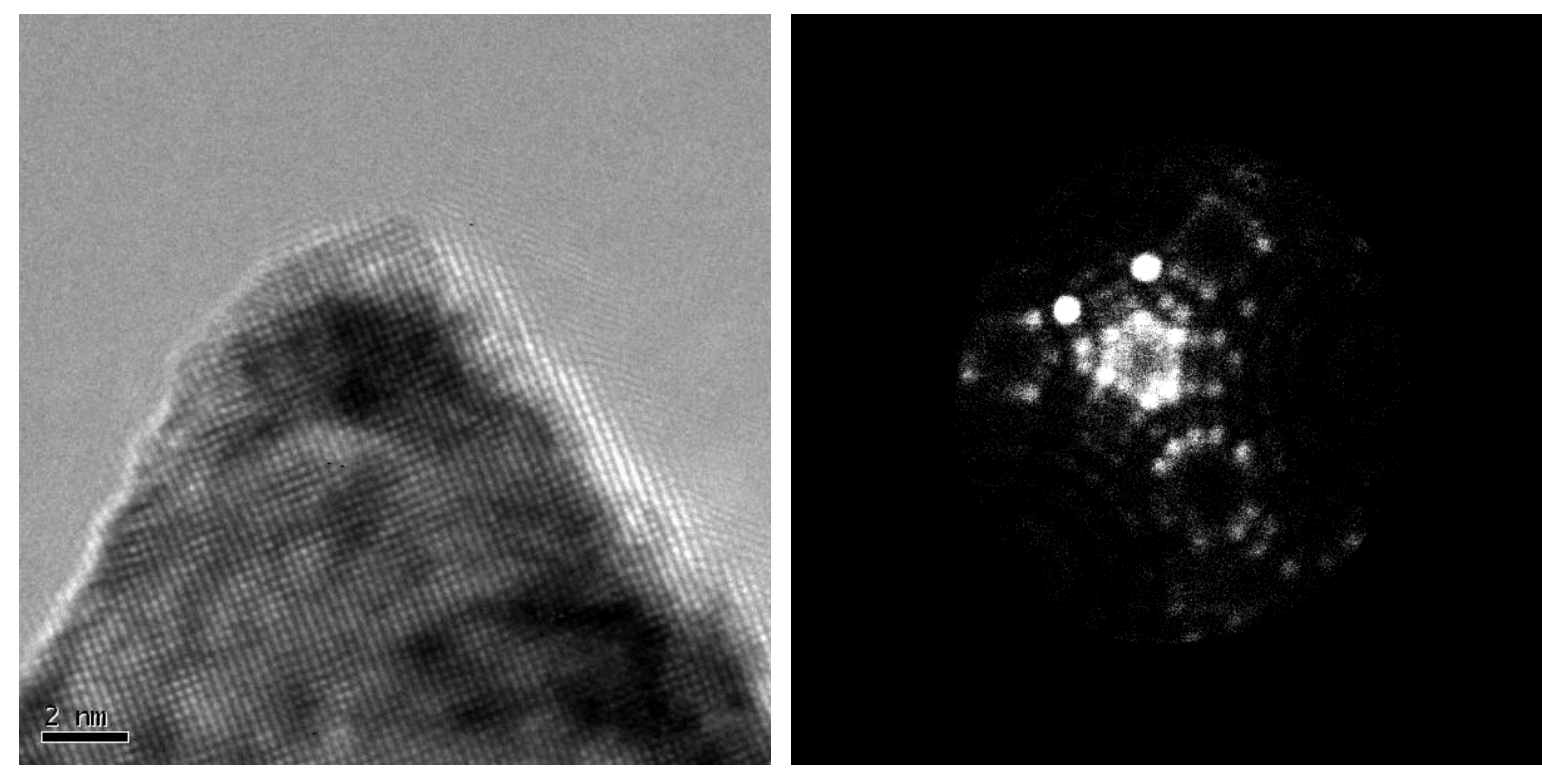

FIG. 2. (a) HRTEM image of a properly handled W tip showing no evidence of oxide formation. (b) Representative field ion microscopy image of a properly handled $\mathrm{W}$ tip showing excellent crystallinity, a strong $<111>$ orientation, and small radius of curvature. 